\title{
Title:
}

\section{TMEM16A overexpression indicates poor prognosis in colorectal cancer patients}

\section{Authors:}

Jia-Jia Liu, Fang He, Shi-Bin Guo

DOI: $10.17235 /$ reed.2021.8292/2021

Link: PubMed (Epub ahead of print)

Please cite this article as:

Liu Jia-Jia, He Fang, Guo Shi-Bin. TMEM16A overexpression indicates poor prognosis in colorectal cancer patients. Rev Esp Enferm Dig 2021. doi: 10.17235/reed.2021.8292/2021.

This is a PDF file of an unedited manuscript that has been accepted for publication. As a service to our customers we are providing this early version of the manuscript. The manuscript will undergo copyediting, typesetting, and review of the resulting proof before it is published in its final form. Please note that during the production process errors may be discovered which could affect the content, and all legal disclaimers that apply to the journal pertain. 
TMEM16A overexpression indicates poor prognosis in colorectal cancer patients

Jia-Jia Liu ${ }^{1,2}$, Fang $\mathrm{He}^{1,3}$, Shi-Bin Guo

${ }^{1}$ Department of Gastroenterology, The First Affiliated Hospital, Dalian Medical University, Dalian, Liaoning 116011

${ }^{2}$ Department of Gastroenterology, LinFen central hospital, Linfen, Shanxi, 041000

${ }^{3}$ Department of Gastroenterology, Cadre ward, Gansu Provincial People's Hospital, Lanzhou, Gansu, 730000

Correspondence to: Professor Shi-Bin Guo, Department of Gastroenterology, The First Affiliated Hospital, Dalian Medical University, 222 Zhongshan Road, Dalian, Liaoning 116011, P.R. China

E-mail:1440507916@qq.com

\section{Abstract}

Aim: To investigate the relationship between TMEM16A and the clinicopathological features and prognosis of patients with colorectal cancer (CRC).

Methods: 96 patients with CRC that were confirmed by pathology after being operated on at the First Affiliated Hospital of Dalian Medical University between June 2009 and December 2011 were enrolled and followed up upon. The expression of TMEM16A protein in CRC was detected by immunohistochemistry in 96 cases. The relationship between the expression of TMEM16A protein in CRC and the clinical features and clinical prognosis were analyzed.

Results: 1 . There was no correlation between the TMEM16A protein expression and gender, age, or tumor location, size, and degree of differentiation $(P>0.05)$. However, the expression of TMEM16A protein was significantly associated with the depth of invasion, lymph node metastasis, and the Dukes stage $(P<0.05) .2$. Kaplan-Meier survival curves showed that CRC patients with high expressions of 
TMEM16A protein had poorer overall survival compared with those having low expression levels (68.2\% vs. 92.3\%, $\left.\mathrm{X}^{2}=9.892, \mathrm{P}=0.002\right)$. Multivariate Cox regression analysis showed that upregulation of TMEM16A protein expression is an independent predictive factor for poor prognosis in patients with $C R C(P<0.05$, $\mathrm{RR}=6.467,95 \% \mathrm{Cl}:$ 1.777- 23.538).

Conclusions: The expression of TMEM16A protein in CRC was associated with tumor invasion, lymph node metastasis, and the Dukes stage. High expression of TMEM16A protein in CRC can be identified as an independent predictive factor for the poor prognosis of patients with CRC.

Key words: Colorectal cancer. TMEM16A. Clinical-pathologic factors. Prognosis.

INTRODUCTION:

Colorectal cancer (CRC) is common worldwide and remains one of the most common causes of cancer-related deaths worldwide[1]. More than 945000 new cases of CRC are diagnosed every year, with 492000 dying annually[2, 3]. Although treatments have improved in recent years, invasion and metastasis are still regarded as the most important factors for the poor prognosis in these patients. However, knowledge of the molecular mechanisms regarding the invasion and migration of CRC are still limited.

TMEM16A (also called DOG1 (Discovered On Gastrointestinal stromal tumors protein 1), ANO1 (ANOCTAMIN-1), ORAOV2 (Oral cancer Overexpressed 2) and TAOS 2 (Tumor-Amplified and Overexpressed Sequence 2)[4-8] is expressed in various tissues, including secretory epithelia, smooth muscle, sensory neurons and other tissues[9]. It plays many important physiological roles, such as control of epithelial fluid transport, vascular smooth muscle contraction, saliva production, and gastrointestinal tract motility[10-12].

Recently, TMEM16A has been reported to be overexpressed in several malignant tumors, including head and neck squamous cell carcinoma (HNSCC), esophageal, 
breast and prostate cancers. Overexpression of TMEM16A is also related to distant metastasis and poor prognosis of cancer patients with $\operatorname{HNSCC}[13,14]$, but its expression and function in CRC is still unclear. In this study, we investigated the biological function of TMEM16A in CRC and the relationship between TMEM16A and the prognosis of these patients.

\section{MATERIALS AND METHODS}

\section{Patients}

This retrospective study was performed in the First Affiliated Hospital of Dalian Medical University. A total of 96 colorectal cancer tissues from patients with CRC who had received radical colorectal resection from June 2009 to December 2011 were included. Exclusion criteria were: Patients having gone through chemotherapy or other antitumor therapy before surgery, patients having other malignant tumors, the clinical data is not complete. The study was executed according to the Helsinki Declaration and followed the local legislation and was approved by the Ethics Committee of the First Hospital affiliated to Dalian Medical University. All the patients or their relatives presented written informed consent before the procedure. Clinicopathological features including gender, age, tumor location, tumor size, tumor differentiation, depth of tumor invasion, lymph node metastasis, Dukes stage, radiotherapy or chemotherapy after operation, and survival time were all recorded. The median follow-up duration was 80 months (range: 17- 96 months).

\section{Reagents}

Mouse anti-human ANO1 polyclonal antibody (Elabscience Biological Technology Co., Ltd. Wuhan, China), anti-mouse IgG (MaxVisionTM2, Maixin Biotechnology, Fuzhou, China), and DAB Substrate kit (Maixin Biotechnology, Fuzhou, China), PBS (0.01M , pH7.2-7.4 ) (Zhongshan Jinqiao Biological Technology, Beijing, China).

Immunohistochemical analysis

Colorectal cancer tissues were embedded in paraffin wax and cut into sections. The sections underwent deparaffinization, rehydration, and inactivation, and were 
incubated with Mouse anti-human ANO1 antibody (1:50), at room temperature for $60 \mathrm{~min}$, and then with a secondary antibody (MaxVisionTM2) at room temperature for $20 \mathrm{~min}$. The primary antibody was replaced by phosphate-buffered saline to serve as a negative control. Five high-power microscopic fields were randomly chosen per slide and the yellow material in the cytoplasm or cell membrane was considered to represent a TMEM16A-positive cell. Cell staining was assigned 4 scores: 4: > 75\% positive cells, 3 : $50 \%-75 \%$ positive cells, $2: 25 \%-50 \%$ positive cells, and 1: $<25 \%$ positive cells. Cell staining intensity was scored based on its color as follows: 0: no staining, 1: faint yellow, 2: light brown, and 3: dark brown. TEME 16 A protein expression was examined by two pathologists with blindness to the clinical data of the patients. The final score was defined as staining intensity $\times$ percentage of positive cells[15]. Score of 8 to 12 was regarded as high expression and score of 0 to 7 was regarded as low expression[16, 17].

\section{Statistical analysis}

Data analysis was performed using the SPSS 17.0 software (Chicago, IL, USA). Overall survival was defined as the time from surgery to death or last follow-up. Overall survival according to TMEM16A protein expression was plotted with the Kaplan-Meier method and the difference was analyzed with the log-rank test. $\mathrm{P}<$ 0.05 was considered statistically significant. Univariate Cox was used to analyze the related factors of poor prognosis of colorectal cancer. Multivariate Cox regression was used to analyze whether the high expression of TMEM16A protein was an independent prognostic factor of poor prognosis in patients with colorectal cancer.

\section{RESULTS}

\section{Baseline characteristics of CRC patients}

96 patients included 58 males and 38 females, aged $30-80$ years old, with a median age of 62.2years; 13 cases were well-differentiated, 61 cases were moderately differentiated, and 22 cases were poorly differentiated; lymph node metastasis was found in 33 cases, with 63 cases having no lymph node metastasis; 6 patients were Dukes stage A, 57 Dukes stage B and 33 patients Dukes stage $C$. 
2. Immunohistochemical staining: The immunohistochemical results showed that TMEM16A protein was mainly located in the cytoplasm and cell membrane of the CRC cells, which is consistent with previous studies[18]. Among the 96 cases, 44 cases (45.8\%) had high expression of TMEM16A protein, and 52 cases (54.2\%) had low expression.

3. The relationship between TMEM16A protein expression and clinicopathological features in CRC patients: The 96 CRC cases were divided into a high expression of TMEM16A protein group and low expression group in accordance with the immunohistochemical evaluation criteria. There was no correlation between the TMEM16A protein expression and gender, age, tumor location, size, and degree of differentiation $(P>0.05)$. However, the expression of TMEM16A protein was significantly associated with the depth of invasion, lymph node metastasis, and Dukes stage $(P<0.05)$. (Table 1$)$.

\section{The relationship between TMEM16A protein expression and clinical prognosis:} The overall survival time of CRC patients in the high TMEM16A expression group was $70.523 \pm 4.569$ (Months), while in low expression group was $86.519 \pm 1.711$ (Months). The Kaplan-Meier survival model showed that the survival time of CRC patients in the high TMEM16A protein expression group was significantly lower than that in the low TMEM16A protein expression group $\left(68.2 \%\right.$ vs. $92.3 \%, X^{2}=9.892, P=$ 0.002) (Figure 1). Univariate analysis showed that the prognosis of CRC patients did not correlate with gender $(P=0.767)$, tumor size $(P=0.084)$, and tumor differentiation $(P=0.557)$ and invasive depth $(P=0.827)$, but correlates with age $(R R=5.671,95 \%$ confidence interval $(\mathrm{Cl})$ : $1.303-24.678, \mathrm{P}<0.05)$, lymph node metastasis ( $R R=3.645$, 95\% Cl: 1.411-9.415, $\mathrm{P}<0.05)$, Dukes stage ( $\mathrm{RR}=3.645,95 \% \mathrm{Cl}$ : 1.411-9.415, $\mathrm{P}<0.05)$, the expression of TMEM16A (RR=4.972, 95\% Cl: $1.635-15.120, P<0.05)$ and postoperative radiochemotherapy $(\mathrm{RR}=1.659,95 \% \mathrm{Cl}: 1.016-2.708, \mathrm{P}<0.05)$. Multivariate Cox regression analysis showed that upregulation of TMEM16A protein expression was an independent predictive factor for the poor prognosis of patients with CRC ( $P<0.05, R R=6.467,95 \% \mathrm{Cl}: 1.777-23.538)$. 


\section{DISCUSSION:}

TMEM16A, regarded as a calcium-activated chloride channel ( $\mathrm{CaCC})$, has been reported closely associated with some malignant tumors in previous studies, including CRC. Sui etc.[19] reported that TMEM16A was over-expressed in several colorectal cancer cells, but little is reported about the significance of the high expression of TMEM16A in CRC patients.

Our study showed that there was no correlation between the TMEM16A protein expression and gender, age, tumor location, size, or degree of differentiation. However, high expression of TMEM16A protein was significantly associated with deeper invasion, lymph node metastasis, and higher Dukes stage. Our study indicated that TMEM16A was involved in the migration and invasion of CRC. Moreover, Sui etc.[19] reported that over expression of TMEM16A was associated with increased risk of metastasis in some CRC cell lines, and that knockdown of TMEM16A leads to suppression of growth, migration and invasion of CRC cells in vitro experiments. All this evidence indicates that TMEM16A may play an important role in the metastasis of CRC, thereby indicating a basis for TMEM16A as a potential drug target for treating metastatic CRC.

Right-side colon cancers and left-side colon cancers might develop by distinct biological pathways[20]. But in our study, there was no correlation between the TMEM16A protein expression and tumor location. In addition, degree of differentiation is associated with prognosis of CRC patients[21], but there was no correlation between the TMEM16A protein expression and differentiation degree of CRC in our study. These two results are different from the literature, which attributed to the statistical bias caused by small samples.

Despite some advances in the treatment of colorectal cancer, metastasis remains challenging. It is reported that immune-based therapy maybe improve prognosis of patients with lymph nodes metastasis[22]. However, immunotherapy is rarely applied to CRC because of difficulty in identifying new targets. In our study, we also found that CRC patients with high expression of TMEM16A protein had a poorer overall survival rate compared with those with low expression levels of TMEM16A protein, and multivariate Cox regression analysis showed that upregulation of 
TMEM16A protein expression was an independent predictive factor for the poor prognosis of patients with CRC. These can also support the conjecture that high expression of TMEM16A plays an important role in the migration and invasion of CRC. Therefore, TMEM16A may become a potential target for the treatment of CRC. By now, the mechanism by which TMEM16A affects the invasion and migration of CRC remains unclear. It has been reported that ERK pathways may contribute to tumor cell proliferation, migration, invasion, and progression[23-25]. U0126, an inhibitor of ERK, can inhibit the function of TMEM16A[26]; on the other hand, knockdown of TMEM16A significantly inhibits the activation of MEK and ERK1/2[19]. It may be by the ERK pathway that TMEM16A contributes to the progression of CRC. Other studies report that TMEM16A contributes to cancer progression by activating the mitogen-activated protein kinase (MAPK) signaling pathway[27]. By activating epithelial growth factor receptor (EGFR) and calmodulin-dependent protein kinase II (CAMK II) signaling, TMEM16A can promote cancer progress in breast cancer and HNSCC[28], subsequently inducing the activation of AKT and MAPK signaling[27]. However, the molecular mechanism by which TMEM16A affects the invasion and migration of CRC is still not fully understood. Since the poor understanding of the molecular mechanism by which TMEM16A affects invasion and migration of CRC has limited the therapies for CRC, further studies are called for in the future.

However, there are some limitations in this study, such as it is a retrospective study, and the sample size is small, which may cause statistical bias.

In conclusion, TMEM16A is involved in the migration and invasion of CRC. High expression of TMEM16A protein is significantly associated with deeper invasion, lymph node metastasis, and higher Dukes stage. Moreover, CRC patients with high expression of TMEM16A protein had a poorer overall survival rate compared to those with low expression of TMEM16A protein, and up regulation of TMEM16A protein expression can be identified as an independent predictive factor for poor prognosis in patients with CRC. 


\section{REFERENCE}

1. Jemal A, Bray F, Center MM et al: Global cancer statistics. CA Cancer J Clin 2011, 61(2):69-90.

2. Weitz J, Koch M, Debus J et al: Colorectal cancer. Lancet 2005, 365(9454):153-165.

3. Siegel RL, Miller KD, Jemal A: Cancer statistics, 2018. CA Cancer J Clin 2018, 68(1):7-30.

4. Huang X, Godfrey TE, Gooding WE et al: Comprehensive genome and transcriptome analysis of the 11q13 amplicon in human oral cancer and synteny to the 7F5 amplicon in murine oral carcinoma. Genes Chromosomes Cancer 2006, 45(11):1058-1069.

5. Katoh $M$, Katoh $M$ : Identification and characterization of TMEM16E and TMEM16F genes in silico. Int J Oncol 2004, 24(5):1345-1349.

6. Sheridan JT, Worthington EN, Yu K et al: Characterization of the oligomeric structure of the $\mathrm{Ca}(2+)$-activated $\mathrm{Cl}$ - channel Ano1/TMEM16A. J Biol Chem 2011, 286(2):1381-1388

7. Chenevert J, Duvvuri U, Chiosea S et al: DOG1: a novel marker of salivary acinar and intercalated duct differentiation. Mod Pathol 2012, 25(7):919-929.

8. Bulley S, Neeb ZP, Burris SK et al: TMEM16A/ANO1 channels contribute to the myogenic response in cerebral arteries. Circ Res 2012, 111(8):1027-1036.

9. Ferrera L, Caputo A, Galietta L: TMEM16A protein: a new identity for Ca(2+)-dependent Cl(-) channels. Physiology (Bethesda) 2010, 25(6):357-363.

10. Rock JR, O'Neal WK, Gabriel SE et al: Transmembrane protein 16A (TMEM16A) is a Ca2+-regulated $\mathrm{Cl}$ - secretory channel in mouse airways. J Biol Chem 2009, 284(22):14875-14880.

11. Romanenko VG, Catalan MA, Brown DA et al: Tmem16A encodes the Ca2+activated $\mathrm{Cl}$ - channel in mouse submandibular salivary gland acinar cells. J Biol Chem 2010, 285(17):12990-13001.

12. Dutta AK, Khimji AK, Kresge $\mathrm{C}$ et al: Identification and functional characterization of TMEM16A, a Ca2+-activated Cl- channel activated by 
extracellular nucleotides, in biliary epithelium. J Biol Chem 2011, 286(1):766-776.

13. Liu W, Lu M, Liu B et al: Inhibition of $\mathrm{Ca}(2+)$-activated $\mathrm{Cl}(-)$ channel AN01/TMEM16A expression suppresses tumor growth and invasiveness in human prostate carcinoma. Cancer Lett 2012, 326(1):41-51.

14. Carneiro A, Isinger A, Karlsson A et al: Prognostic impact of array-based genomic profiles in esophageal squamous cell cancer. BMC Cancer 2008, 8:98.

15. Guo SB, Duan ZJ, Li Q et al: Effect of heme oxygenase-1 on renal function in rats with liver cirrhosis. World J Gastroenterol 2011, 17(3):322-328.

16. Lin $\mathrm{Y}$, Zhai $\mathrm{H}$, Ouyang $\mathrm{Y}$ et al: Knockdown of PKM2 enhances radiosensitivity of cervical cancer cells. Cancer Cell Int 2019, 19:129.

17. Sheng N, Yan L, Wu K et al: TRIP13 promotes tumor growth and is associated with poor prognosis in colorectal cancer. Cell Death Dis 2018, 9(3):402.

18. Thomas-Gatewood C, Neeb ZP, Bulley S et al: TMEM16A channels generate $\mathrm{Ca}(2)(+)$-activated $\mathrm{Cl}(-)$ currents in cerebral artery smooth muscle cells. Am J Physiol Heart Circ Physiol 2011, 301(5):H1819-1827.

19. Sui $Y$, Sun $M$, Wu $F$ et al: Inhibition of TMEM16A expression suppresses growth and invasion in human colorectal cancer cells. PLOS One 2014, 9(12):e115443.

20. Liu $\mathrm{F}$, Li C, Jia $\mathrm{H}$ et al: Is there a prognostic value of tumor location among Chinese patients with colorectal cancer? Oncotarget 2017, 8(24):38682-38692.

21. Vasile L, Olaru A, Munteanu M et al: Prognosis of colorectal cancer: clinical, pathological and therapeutic correlation. Rom J Morphol Embryol 2012, 53(2):383-391.

22. Argentiero A, De Summa S, Di Fonte R et al: Gene Expression Comparison between the Lymph Node-Positive and -Negative Reveals a Peculiar Immune Microenvironment Signature and a Theranostic Role for WNT Targeting in Pancreatic Ductal Adenocarcinoma: A Pilot Study. Cancers (Basel) 2019, 11(7). 
23. Jin Q, Liu G, Domeier PP et al: Decreased tumor progression and invasion by a novel anti-cell motility target for human colorectal carcinoma cells. PLOS One 2013, 8(6):e66439.

24. Peng $C$, Liu HY, Zhou $M$ et al: BRD7 suppresses the growth of Nasopharyngeal Carcinoma cells (HNE1) through negatively regulating betacatenin and ERK pathways. Mol Cell Biochem 2007, 303(1-2):141-149.

25. Chen $\mathrm{H}$, Zhu G, Li Y et al: Extracellular signal-regulated kinase signaling pathway regulates breast cancer cell migration by maintaining slug expression. Cancer Res 2009, 69(24):9228-9235.

26. Almaca J, Tian $\mathrm{Y}$, Aldehni $\mathrm{F}$ et al: TMEM16 proteins produce volumeregulated chloride currents that are reduced in mice lacking TMEM16A. J Biol Chem 2009, 284(42):28571-28578.

27. Duvvuri U, Shiwarski DJ, Xiao D et al: TMEM16A induces MAPK and contributes directly to tumorigenesis and cancer progression. Cancer Res 2012, 72(13):3270-3281.

28. Britschgi A, Bill A, Brinkhaus $\mathrm{H}$ et al: Calcium-activated chloride channel ANO1 promotes breast cancer progression by activating EGFR and CAMK signaling. Proc Natl Acad Sci U S A 2013, 110(11):E1026-1034.

Table1. The relationship between TMEM16A protein expression and clinicopathological features of colorectal cancer 


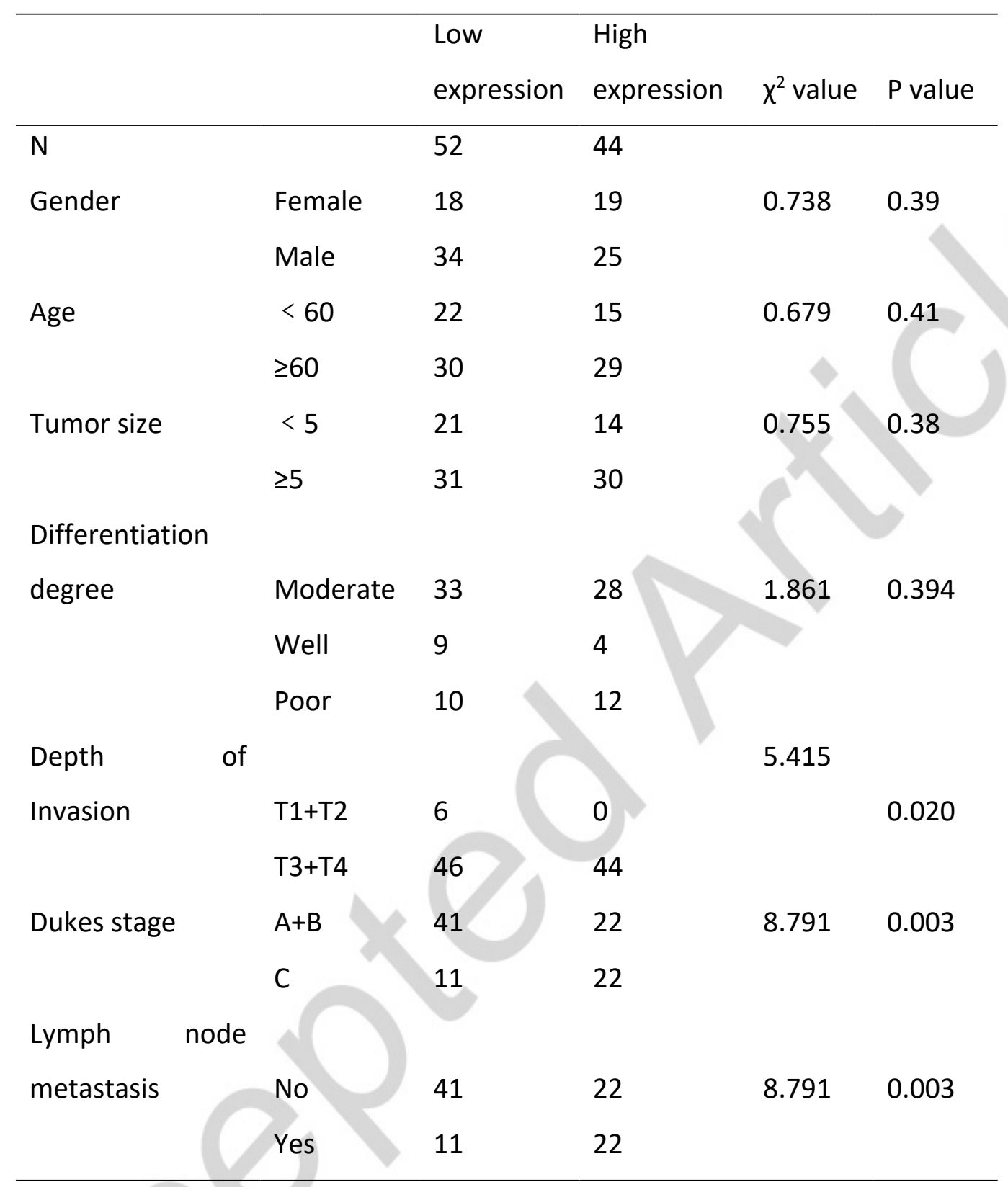




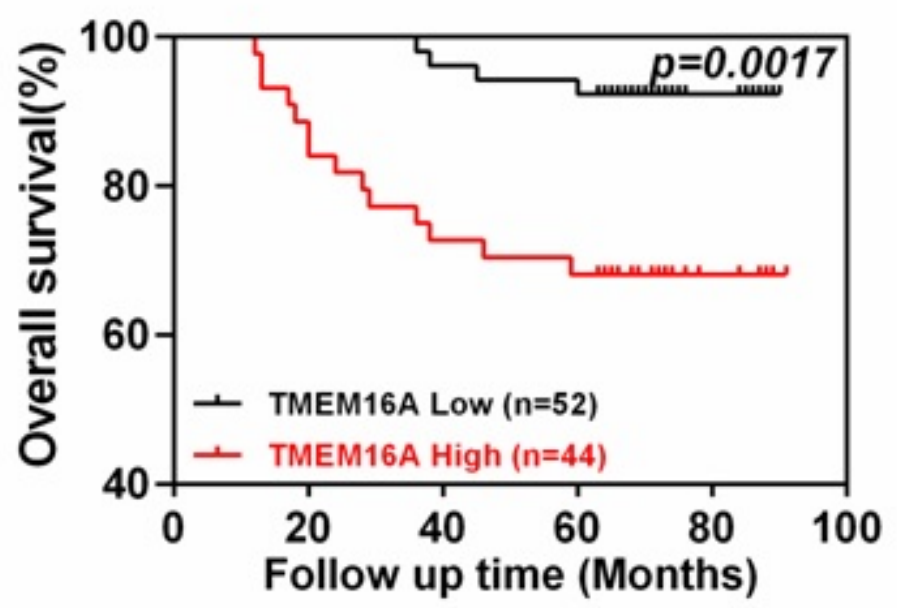

Fig1. Kaplan-Meier analysis of overall survival for colorectal cancer patients according to the expression level of TMEM16A protein.

\section{Conflict of interest}

The authors declare that they have no conflicts of interest.

\section{Author contributions}

GUO shibin designed the study; LIU jiajia and HE fang collected and analyzed the data; GUO shibin, LIU jiajia and HE fang wrote the manuscript; LIU jiajia and HE fang have equal contributions. All the authors read and approved the final manuscript. 